\title{
Generation of short-pulse VUV and XUV radiation
}

\author{
B. WELLEGEHAUSEN, H. WELLING, C. MOMMA, \\ M. FEUERHAKE, K. MOSSAVI, H. EICHMANN \\ Institut für Quantenoptik, Universität Hannover, Welfengarten 1, 30167 \\ Hannover, Germany
}

Received 26 September 1994; revised and accepted 10 April 1995

Starting from intense short-pulse KrF $(248 \mathrm{~nm}, 25 \mathrm{~mJ}, 400 \mathrm{fs})$, ArF $(193 \mathrm{~nm}, 10 \mathrm{~mJ}$, $\sim 1 \mathrm{ps}$ ), and Ti:sapphire $(810 \mathrm{~nm}, 100 \mathrm{~mJ}, 150 \mathrm{fs}$ ) laser systems, schemes for the generation of fixed-frequency and tunable VUV and XUV radiation by nonlinear optical techniques are investigated. With the $\mathrm{KrF}$ system, a four-wave mixing process in xenon yields tunable radiation in the range of $130-200 \mathrm{~nm}$ with output energies of, so far, $100 \mu \mathrm{J}$ in less than $1 \mathrm{ps}$. For the XUV spectral range below $100 \mathrm{~nm}$, nonperturbative high-order harmonic generation and frequency mixing processes in noble gas jets are considered. To achieve tunability, the intense fixed-frequency pump laser radiation is mixed with less intense but broadly tunable radiation from short-pulse dye lasers or optical parametric generator-amplifier systems. In this way, tunability down to wavelengths of less than $40 \mathrm{~nm}$ has been demonstrated.

\section{Introduction}

The generation of coherent radiation in the VUV spectral range below $200 \mathrm{~nm}$ or in the XUV range starting below $100 \mathrm{~nm}$ is of interest for a variety of potential scientific and technical applications [1]. Although great efforts have been and are still being undertaken to develop primary laser sources in this spectral range, only very few laboratory soft $\mathrm{x}$-ray laser systems exist so far $[1,2]$, and the shortest wavelengths of commercial VUV systems are still the discharge-pumped ArF $(193 \mathrm{~nm})$ and $F_{2}(157 \mathrm{~nm})$ excimer lasers. Even if more laboratory laser systems at short wavelengths become available in the future, these systems will not be tunable, which will limit scientific use in particular. In order to cover broad spectral ranges in the VUV and XUV, therefore, nonlinear optical techniques have to be used, and as a result of recent progress in the realization of compact high-peak-power pump laser systems, interesting new perspectives for these techniques have been developed.

With conventional nanosecond pump sources and power levels typically in the megawatt range, only third-order and fifth-order nonlinear processes are of practical interest. Using four-wave sum- and difference-frequency mixing schemes and favourable two-photon resonances, tunable radiation down to about $70 \mathrm{~nm}$ has been achieved with output power levels in the $1 \mathrm{~W}$ range at wavelengths below $100 \mathrm{~nm}$ [3-6]. These sources are well developed and widely used, mostly for linear spectroscopic experiments [7]. Recently experiments have been reported with narrowband tunable radiation around $58 \mathrm{~nm}$ generated by fifth-order 


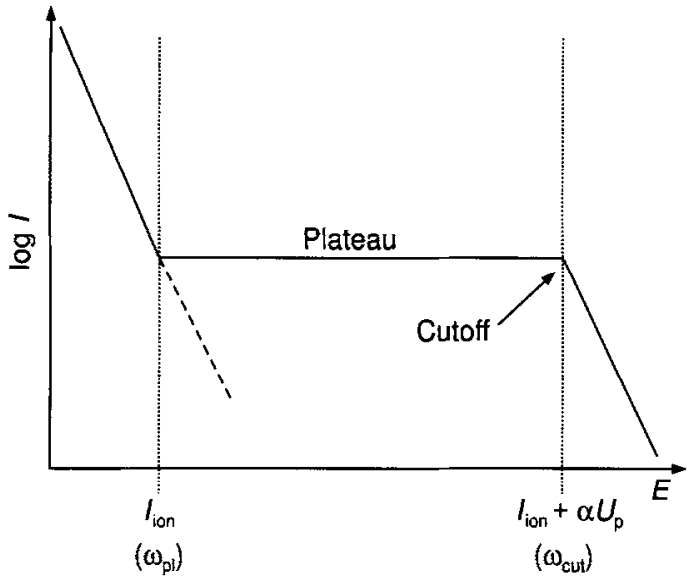

Figure 1 Schematic trend of a harmonic power spectrum, with plateau and cutoff. In perturbative nonlinear optics the harmonic power decreases continuously (dashed line).

processes [8]. To reach shorter wavelengths and also higher output powers, higher-order nonlinear processes and much higher peak powers of the pump sources have to be used. Systems suitable for this are short-pulse ( $\mathrm{ps}-\mathrm{fs}$ ) laser systems at gigawatt power levels, which, when focused, allow intensities well above $10^{13} \mathrm{~W} \mathrm{~cm}^{-2}$. At these intensities, corresponding to electric field strengths above $10^{8} \mathrm{~V} \mathrm{~cm}^{-1}$, the optical interaction is no longer a small perturbation, resulting in a dramatic change of the nonlinear response as indicated by the schematic diagram of a typical harmonic power spectrum in Fig. 1. After an initial steep (perturbative) decrease of the power for the low-order harmonics, a plateau region is reached which extends up to high harmonics, until finally at a characteristic cutoff frequency a strong decrease again follows.

With high-intensity Nd:glass, Ti:sapphire, dye and $\mathrm{KrF}$ excimer laser systems, harmonic generation has been studied [9-12] and harmonics up to orders of 143 and shortest wavelengths around $7 \mathrm{~nm}$ have been demonstrated [13]. Thus, with high-intensity pump sources, new aspects in nonlinear optics open up.

In this contribution different approaches to the generation of powerful and tunable shortpulse radiation in the VUV and XUV spectral ranges will be described and briefly discussed. For the experiments, the commercial $\mathrm{KrF}$ (25 mJ, $400 \mathrm{fs}$; Laser Lab. Göttingen; Lambda Physik) and Ti:sapphire (100 mJ, $150 \mathrm{fs}$; B.M. Industries) laser systems described below werc used. With a near-resonant four-wave mixing process in xenon [14], tunable subpicosecond radiation in the range of about $130-200 \mathrm{~nm}$ can be generated with output powers exceeding $100 \mu \mathrm{J}$; and using high-order mixing processes, tunability down to less than $40 \mathrm{~nm}$ has been demonstrated.

\section{Pump laser systems}

The short-pulse pump laser systems at $248 \mathrm{~nm}(\mathrm{KrF})$ and at about $810 \mathrm{~nm}$ (Ti:sapphire) are shown schematically in Figs 2 and 3 . In both systems, short pulses generated by suitable oscillators are amplified to higher energies.

The excimer system (Fig. 2) consists of a standard double-discharge tube excimer laser (EMG 150; Lambda Physik), in which one discharge channel is operated as a 20-ns oscillator at $308 \mathrm{~nm}(\mathrm{XeCl})$ and the other as an amplifier at $248 \mathrm{~nm}(\mathrm{KrF})$. Because excimer lasers cannot directly generate short pulses, femtosecond pulses at $496 \mathrm{~nm}(2 \times 248 \mathrm{~nm})$ are first generated by a specially designed dye laser chain [15], pumped by the $308 \mathrm{~nm}$ radiation from the $\mathrm{XeCl}$ 


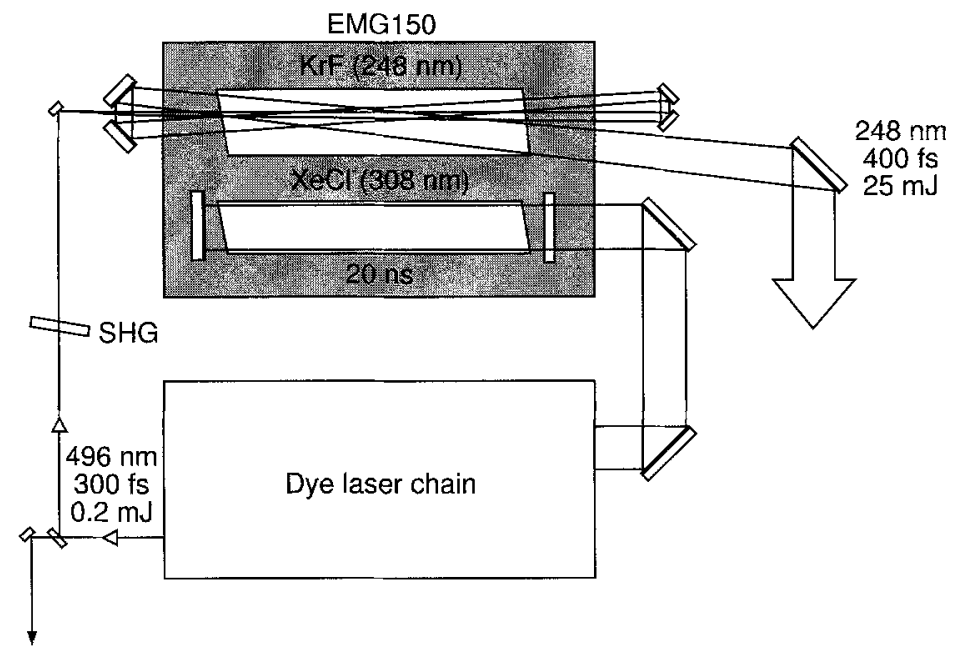

Figure 2 Short-pulse KrF excimer laser system.

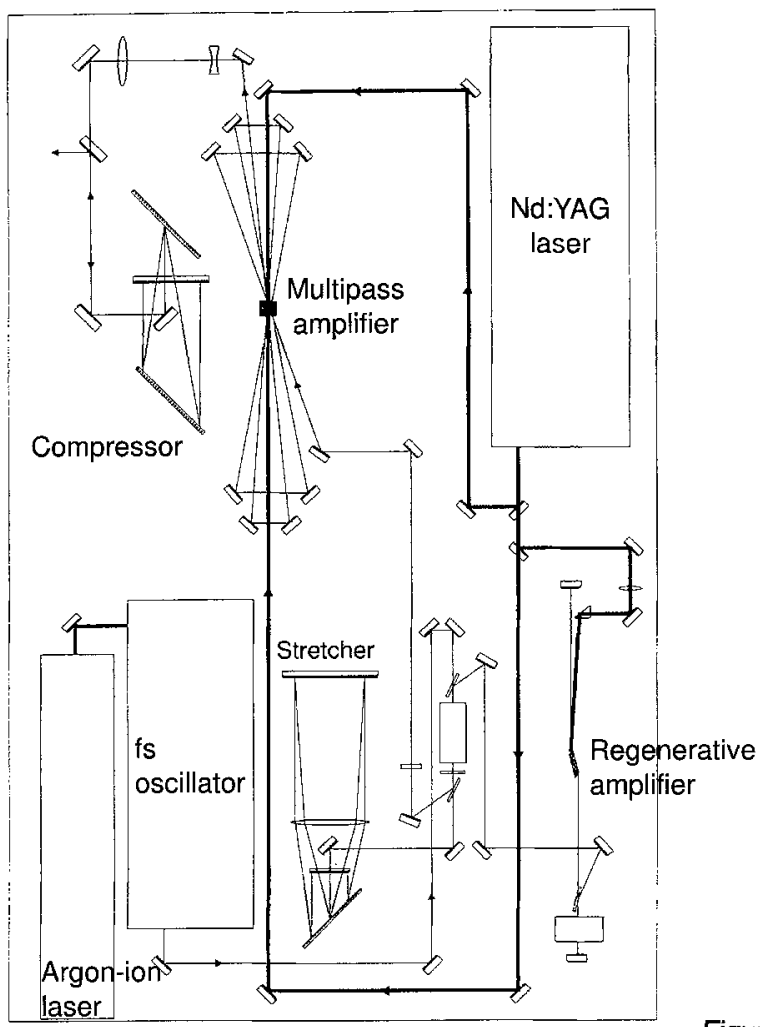

Figure 3 High-power Ti:sapphire laser system. 
oscillator. This chain finally delivers at $496 \mathrm{~nm}$ pulses with a duration of $300 \mathrm{fs}$ at an energy of about $200 \mu \mathrm{J}$. After frequency doubling, $248 \mathrm{~nm}$ pulses are obtained which are then amplified in the amplifier channel. With a three-pass off-axis amplification geometry [16], pulses with an energy up to $25 \mathrm{~mJ}$ in about $400 \mathrm{fs}$ and a quadratic beam shape $\left(\sim 3 \times 3 \mathrm{~cm}^{2}\right.$ at the exit window) are obtained. The pulses are not Fourier limited and may be further compressed [15]. In addition to the $248 \mathrm{~nm}$ radiation, powerful pulses of about $100 \mu \mathrm{J}$ at $496 \mathrm{~nm}$ after the frequency doubler may also be used for nonlinear optical experiments.

The Ti:sapphire laser system uses the chirped-pulse amplification (CPA) technique [13] to generate pulses at the terawatt power level. A CW mode-locked Tisapphire oscillator (Coherent Mira) first generates a continuous train of $150 \mathrm{fs}$ pulses (repetition rate $\sim 80 \mathrm{MHz}$ ) at an average energy of about $1 \mathrm{~W}$. These pulses are stretched to about $200 \mathrm{ps}$ and then a single pulse is subsequently amplified in Ti:sapphire amplifier crystals pumped by the sccond harmonic of a powerful Nd:YAG laser ( $\mathrm{J}, 10 \mathrm{~ns}$, repetition rate $10 \mathrm{~Hz}$ ). Amplification is first performed in a regenerative amplifier up to pulse energies of $\sim 8 \mathrm{~mJ}$ and then in a six-pass amplifier to, presently, about $200 \mathrm{~mJ}$. After final pulse compression the system delivers a beam (diameter $\sim 2 \mathrm{~cm}$ ) with energy up to $100 \mathrm{~mJ}$ in $150 \mathrm{fs}$. The pulses are almost Fourier limited.

\section{Four-wave mixing in xenon}

For the generation of tunable VUV radiation a near-resonant four-wave mixing process in xenon is used [14]. By excitation of xenon with the KrF short-pulse radiation near the $5 p^{6}-$ 6 p two-photon resonance (Fig. 4) at intensities of about $10^{13} \mathrm{~W} \mathrm{~cm}^{-2}$, a strong nonlinear polarization is generated, which can start intense parametric oscillations. For the tunable VUV generation, in addition, a tunable visible to near infrared laser field has to be injected, as indicated in Fig. 4. Phase matching can be accomplished by a noncollinear mixing geometry [14]. With nanosecond tunable pulses in the range of $350 \mathrm{~nm}$ to $2 \mu \mathrm{m}$, tunable subpicosecond VUV radiation in the range of $\sim 200-130 \mathrm{~nm}$ has been demonstrated [17]. Nanosecond pulses can be used to generate short VUV pulses, because the two-photon polarization induced by the $\mathrm{KrF}$ laser is short-living. However, it is more favourable to use subpicosecond tunable radiation directly derived from the main pump system, in order to avoid problems due to the synchronization of independent sources. Therefore, distributed feedback or travelling-wave

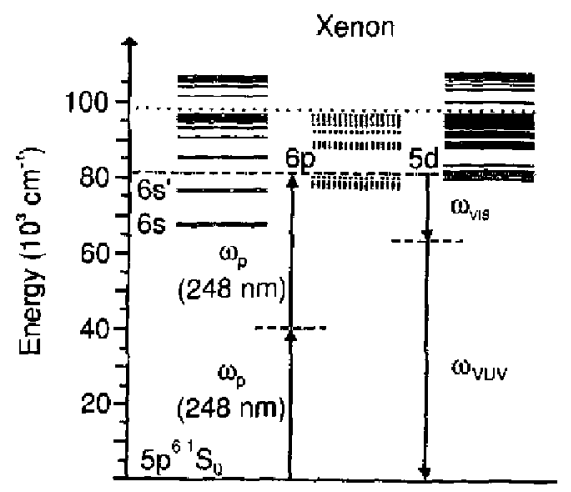

Figure 4 Xenon level scheme with near-resonant parametric four-wave mixing. 
dye lasers, pumped by a fraction of the short-pulse $496 \mathrm{~nm}$ or $248 \mathrm{~nm}$ radiation from the system, have been developed [18], which allow the generation of subpicosecond pulses in the 350$600 \mathrm{~nm}$ range with energies of several microjoules. Figure 5 shows examples where these pulses have been used to generate VUV radiation at wavelengths around $193 \mathrm{~nm}$ and $157 \mathrm{~nm}$, respectively. Pulses at these wavelengths have been used to study the short-pulse amplification characteristics of discharge-excited $A r F(193 \mathrm{~nm})$ and $F_{2}$ excimer amplifiers $[19,20]$. At the ArF wavelength, pulses of about 1 ps with energies up to $10 \mathrm{~mJ}$ could be generated in this way. This radiation has been used in frequency mixing experiments described below.

In the mixing process considered, both radiation fields $\omega_{v_{1 s}}$ and $\omega_{\text {Yuv }}$ are exponentially amplified in the small-signal regime. From investigations of the self-starting four-wave
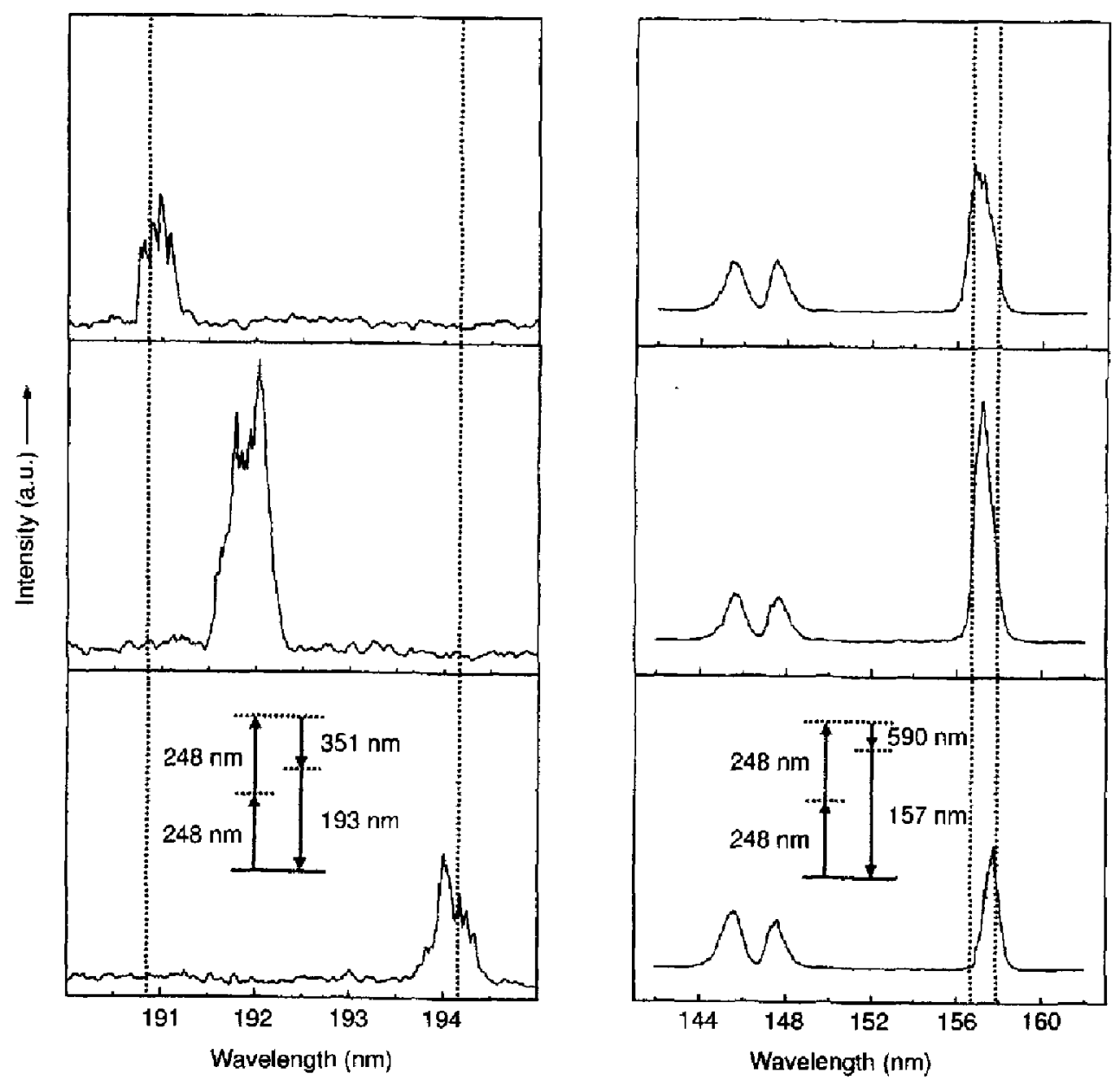

Figure 5 Spectra of generated VUV radiation tuned around $193 \mathrm{~nm}$ and $157 \mathrm{~nm}$. Pump energy $(248 \mathrm{~nm}) 5 \mathrm{~mJ}$ $\left(400 \mathrm{ps}, 10^{13}-10^{14} \mathrm{~W} \mathrm{~cm}^{-2}\right.$ ). With about $80 \mu \mathrm{J}$ at $351 \mathrm{~nm}(\leq 2.5 \mathrm{ps}$ ) and $3 \mu \mathrm{J}$ at $590 \mathrm{~nm}$ (400 fs), about $\sim 5 \mu \mathrm{l}$ at $193 \mathrm{~nm}(<1 \mathrm{ps})$ and $3 \mu \mathrm{J}$ at $157 \mathrm{~nm}$ (<1 ps) are generated. The emission around $146 \mathrm{~nm}$ is due to a self-starting four-wave parametric oscillation process (see [14]). 


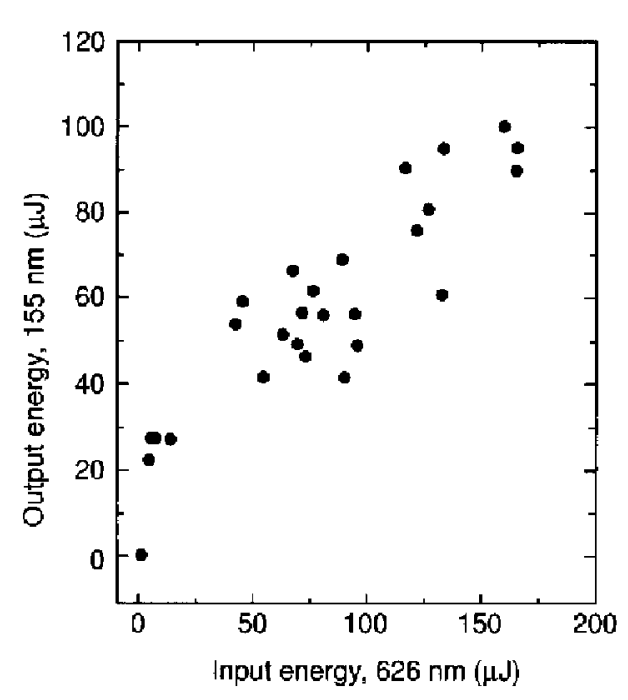

Figure 6 Energy of short-pulse VUV radiation at $155 \mathrm{~nm}$ versus energy of the visible input radiation at $626 \mathrm{~nm}$. Pump energy $(248 \mathrm{~nm})$ is $11 \mathrm{~mJ}$.

parametric generation process in xenon [14] it is known that tremendous amplifications are possible, so that fields with macroscopic output energies can build up from noise, as can be seen from the emission atound $146 \mathrm{~nm}$ in Fig. 5. At higher energies of the fields, the amplification will saturate, and in this regime it can be expected that the energy $E_{\mathrm{VuV}}$ of the VUV field increases proportional to the energy $E_{\mathrm{vin}}$ of the visible input field and reaches comparable values. This can already be seen from the data of Fig. 5. To further increase the VUV energy, mixing experiments at higher energies of the visible input field have been performed, which are summarized in Fig. 6. With up to $180 \mu \mathrm{J}$ input radiation at $626 \mathrm{~nm}$ ( $330 \mathrm{fs}$ ), a maximum VUV energy of $100 \mu \mathrm{J}$ has been achieved so far for about $11 \mathrm{~mJ}$ of pump radiation at $248 \mathrm{~nm}$. As can be seen from Fig. 6, at low $626 \mathrm{~nm}$ input energies the VUV energy increases strongly and then turns over to an almost linear increase. The $626 \mathrm{~nm}$ short-pulse radiation was generated by Raman shifting (first Stokes in hydrogen) of the $496 \mathrm{~nm}$ radiation from the main excimer pump laser system (see Fig. 2) and subsequent amplification in dye solutions pumped by $2 \mathrm{w}$ of a Nd:YAG laser. Because no saturation can be seen in the present experiments, it is expected that short-pulse tunable VUV radiation around $160 \mathrm{~nm}$ with energies approaching the $0.5 \mathrm{~mJ}$ level and gigawatt powers for subpicosecond pulses will be possible, which should be of interest for applications including further nonlinear frequency conversion into the XUV. For the future it is planned to generate the tunable short-pulse input radiation by use of tunable optical parametric generator-amplifier (OPG) schemes.

\section{Fixed frequency harmonic generation}

For the generation of short-pulse radiation in the XUV spectral range, higher-order nonlinear processes have to be used when starting from presently available high-power short-pulse pump laser systems. With $\mathrm{KrF}$ and $\mathrm{ArF}$ lasers, frequency tripling will lead to radiation of $83 \mathrm{~nm}$ and $64 \mathrm{~nm}$ and with fifth-order harmonies $50 \mathrm{~nm}$ and $39 \mathrm{~nm}$ can already be reached, whereas, with Tisapphire lasers at $800 \mathrm{~nm}$, harmonics of the order 9 up to 21 have to be considered. Therefore, intuitively the short-wavelength systems seem to be favoured, which is true in the perturbative regime of nonlinear optics. However, at high pump intensities this situation changes, as indicated by the schematic harmonic response curve in Fig. 1. This nonperturbative 


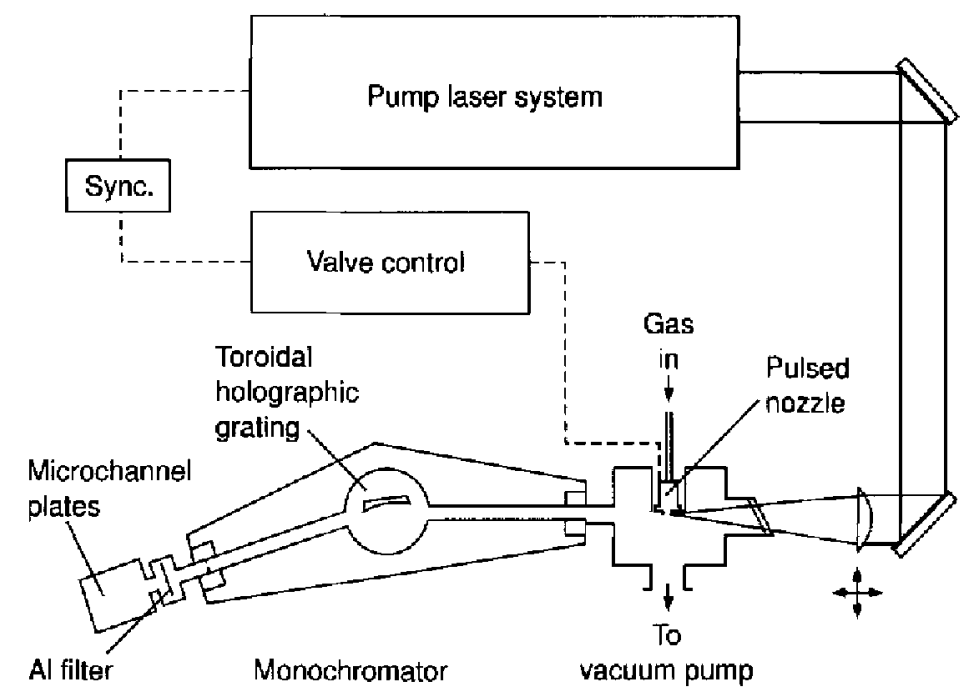

Figure 7 Experimental setup for the generation and detection of XUV radiation.

response is characterized by two frequencies. A lower frequency $\omega_{\mathrm{pl}}$ where the plateau starts, and a cutoff frequency $\omega_{\text {cut }}$ where the plateau ends. The energy $\hbar \omega_{\mathrm{pl}}$ corresponds to the ionization potential $I_{\text {ion }}$ of the nonlinear material, while the cutoff energy is given by $\hbar \omega_{\text {cut }}=I_{\text {ion }}+$ $\alpha U_{\mathrm{p}}[21]$, with $U_{\mathrm{p}}=\left(e^{2} / 2 \epsilon_{0} m_{\mathrm{e}} c\right) I_{\mathrm{p}} / \omega_{\mathrm{p}}^{2}$ being the ponderomotive potential; $\omega_{\mathrm{p}}$ is the pump laser frequency and $I_{\mathrm{p}}$ the pump intensity of the laser up to the limit of the saturation intensity $I_{\text {sat }}$ [22]. Theoretically, the factor $\alpha$ is 3.2 (single-atom response) [23], while experimentally $\alpha$ values in the range of $2-3$ have been observed $[22,24]$. The $1 / \omega_{\mathrm{p}}^{2}$ dependence of the cutoff frequency therefore favours longer-wavelength pump lasers for shorter wavelength generation. However, with shorter-wavelength pump lasers, higher efficiencies at lower orders can be expected.

To test this behaviour, harmonic generation with short-pulse KrF, ArF and Ti:sapphire laser radiation has been compared. The basic experimental setup for this and for frequency mixing experiments described later is shown in Fig. 7. The pump radiation is focused into a gas jet emerging from a pulsed nozzle into a vacuum chamber. The XUV radiation generated is analysed down to about $10 \mathrm{~nm}$ by an XUV monochromator (Yobin Ivon L.HT 30) and detected by a microchannel plate detector (Galileo, $d=3.2 \mathrm{~cm}$ ). For the experiments, noble gases and hydrogen were used. At backing pressures of the nozzle in the range of several bars, vapour pressures in the jet of several tens of millibars are reached, as determined experimentally. The gas jet had a thickness of about $1 \mathrm{~mm}$ at the working distance immediately behind the nozzle exit.

Figures 8 and 9 show examples of harmonic spectra for $\mathrm{KrF}$ in helium and for Ti:sapphire in neon, and Figs 10-12 summarize the data obtained. For ArF only the fifth harmonic could be observed in argon; for $\mathrm{KrF}$ up to the 11th harmonic in helium; and for Ti:sapphire in neon so far the $71 \mathrm{st}$ harmonic at $11 \mathrm{~nm}$, the limit of the detection system. In the literature, for Ti:sapphire and $\mathrm{KrF}$ harmonics up to orders of 109 [10] and 25 [12] have been reported, whereas for ArF no higher harmonics than the fifth have been seen so far [25].

For the interpretation of the dependences, the data and parameters summarized in Table $I$ are helpful. The ionization potential defines the plateau wavelength $\lambda_{\mathrm{pl}}\left(\omega_{\mathrm{pl}}\right)$. With increasing ionization energy, from the heavy to the light noble gases, the polarizability and consequently 

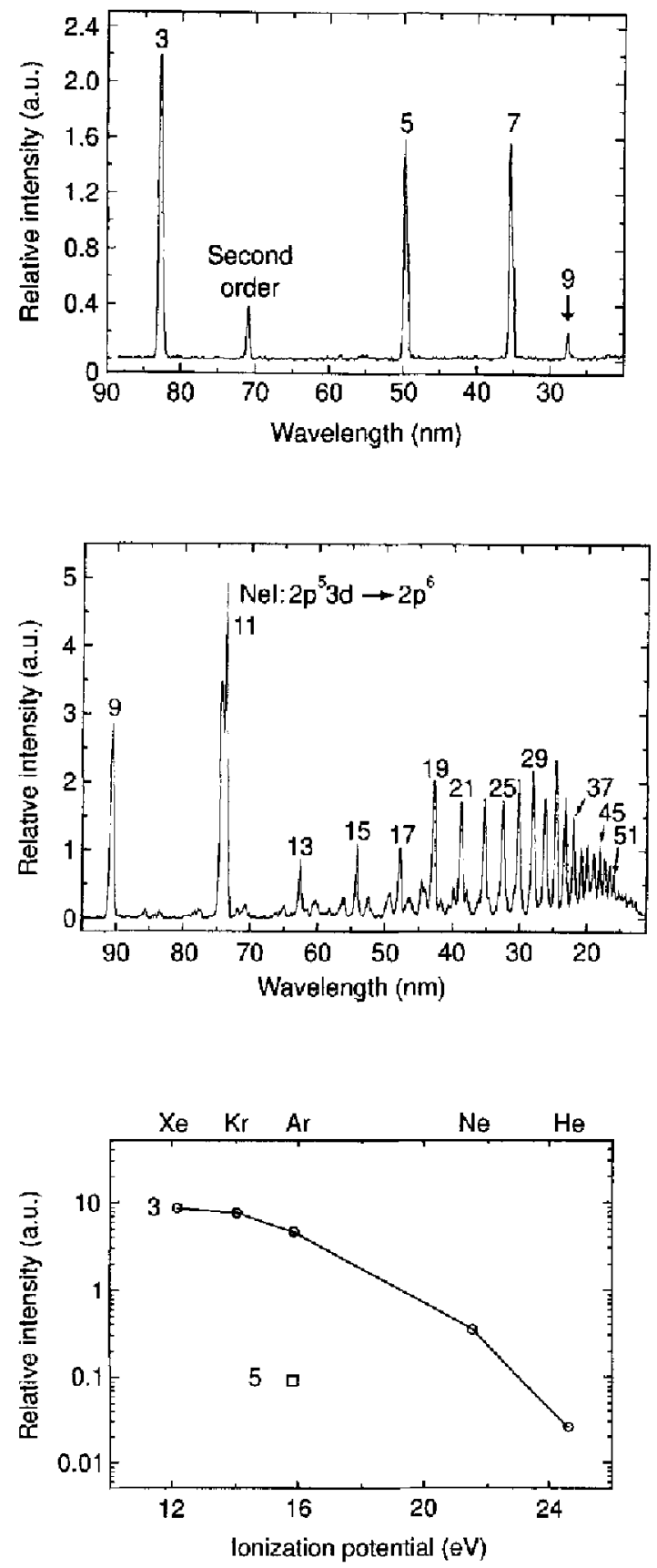

Figure 8 Harmonic spectrum of $\mathrm{KrF}(248 \mathrm{~nm})$ pump radiation in helium. Pump intensity $\sim 10^{16} \mathrm{~W} \mathrm{~cm}^{-2}$. The spectrum is not corrected with respect to the monochromator and detector characteristics.

Figure 9 Harmonic spectrum of Ti:sapphire $(810 \mathrm{~nm})$ pump radiation in neon. The spectrum is not corrected. The signals between the marked harmonics are due to the second grating order of the monochromator.

Figure 10 Intensity of third-and fitth-order harmonics of $193 \mathrm{~nm}$ radiation (ArF, intensity $\sim 10^{15} \mathrm{Wcm}{ }^{2}$ ) for different noble gases (ionization potentials).

the harmonic power decreases. This can be seen clearly from the data in Fig. 10 for the third harmonic of ArF. To see whether a plateau is possible and to estimate the cutoff wavelength $\lambda_{\text {cut }}$, the ponderomotive potential $U_{p}$ has to be determined. As discussed above, the intensity in the equation for $U_{\mathrm{p}}$ is limited by the saturation intensity $l_{\text {sat }}$, which is the intensity where 
TABLE

\begin{tabular}{|c|c|c|c|c|c|c|c|c|c|c|c|c|c|c|c|}
\hline \multirow{2}{*}{$\begin{array}{l}\text { Pump laser: } \\
\text { Noble gas: }\end{array}$} & \multicolumn{5}{|c|}{$\mathrm{KrF}(248 \mathrm{~nm})$} & \multicolumn{5}{|c|}{$\operatorname{ArF}(193 \mathrm{~nm})$} & \multicolumn{5}{|c|}{ Ti:sapphire $(810 \mathrm{~nm})$} \\
\hline & Xe & $\mathrm{Kr}$ & Ar & $\mathrm{Ne}$ & $\mathrm{He}$ & $\mathrm{Xe}$ & $\mathrm{Kr}$ & Ar & $\mathrm{Ne}$ & $\mathrm{He}$ & $\mathrm{Xe}$ & $\mathrm{Kr}$ & $\mathrm{Ar}$ & $\mathrm{Ne}$ & $\mathrm{He}$ \\
\hline$I_{\tan }(\mathrm{cV})$ & 12.1 & 14 & 15.8 & 21.6 & 24.6 & 12.1 & 14 & 15.8 & 21.6 & 24.6 & 12.1 & 14 & 15.8 & 21.6 & 24.6 \\
\hline$I_{\text {sat }}\left(10^{14} \mathrm{~W} \mathrm{~cm}^{-2}\right)$ & 1.2 & 1.4 & 3.6 & 11.4 & 16.7 & 03 & 1.7 & 2 & 6.5 & 12.9 & 1 & 1.4 & 2 & 4.8 & 7 \\
\hline$U_{\mathrm{p}}^{\prime}\left(I_{\mathrm{sat}}\right)(\mathrm{eV})$ & 0.7 & 0.8 & 2.1 & 6.6 & 9.6 & 0.1 & 0.6 & 0.7 & 2.3 & 4.5 & 5.8 & 8.6 & 12.2 & 29.4 & 42.8 \\
\hline$I_{\mathrm{on}}+2 U_{\mathrm{p}}(\mathrm{nm})$ & 92 & 79 & 62 & 36 & 28 & 101 & 82 & 72 & 47 & 37 & 52 & 40 & 31 & 15 & 11 \\
\hline
\end{tabular}

The saturation intensity $I_{\text {sat }}$ was calculated using multiphoton ionizatton for the excimer lasers $(\mathrm{KrF}, \mathrm{ArF})$ and tunnelling ionization for the Ti:sapphire laser.

strong ionization occurs, due to multiphoton or field ionization. Multiphoton ionization is more probable for shorter pump laser wavelengths and lower ionization potentials, comesponding to a Keldysh parameter [26] $\gamma \gg 1$, whereas field ionization is the dominant mechanism for long wavelength lasers and media with high ionization potentials $(\gamma<1)$. Estimated values for $I_{\text {sat }}, U_{\mathrm{p}}\left(I_{\text {sat }}\right)$ and $\lambda_{\text {cut }}$ are given in Table I.

From Table I it can be seen that for the ArF laser the cutoff wavelength for argon as nonlinear medium is below the third harmonic wavelength. Therefore, a plateau, or at least higher harmonics, cannot be expected for the heavier noble gases, which is in agreement with the experimental result that only the fifth harmonic has been detected in argon. Owing to the lower polarizability of the lighter elements neon and helium, the signals at the fifth-order harmonics were too weak to be distinguishable from the background. For the $\mathrm{KrF}$ laser a plateau should be possible for neon and helium as nonlinear medium, but a plateau-like feature could only be seen in helium (Fig. 11), with an estimated cutoff corresponding to an $\alpha$-value of approximately 2 .

Sarukura et al. [12] have observed harmonics up to the 25th order in helium $(9.9 \mathrm{~nm})$ with the $\mathrm{KrF}$ radiation at intensities up to $10^{18} \mathrm{~W} \mathrm{~cm}^{-2}$, which is far above the estimated cutoff wavelength and contradicts our observations. As already pointed out by Krause [21], it is believed that these high harmonics arise from ions produced by the high-intensity laser field.

With the Ti:sapphire system clear plateaux and well-defined cutoffs have been observed (Fig. 12). The energetic positions of the cutoffs for the different noble gases and at pump intensities above the corresponding saturation intensities are in good agreement with the calculated values from Table I, assuming an $\alpha$-value of 2.3 . For the heavier noble gases xenon, krypton and

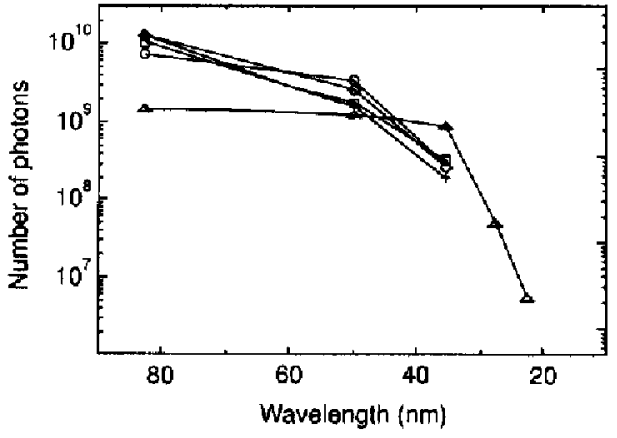

Figure 11 Measured photon numbers of $248 \mathrm{~nm}(\mathrm{KrF}$, $\sim 10^{16} \mathrm{Wcm}^{2}$ ) harmonic radiation for different noble gases: $\diamond$, xenon; + , kryplon; $\square$, argon; 0 , neon; $\Delta$, helium. For the third harmonics the photon numbers correspond to energies of $3-30 \mathrm{~nJ}$ and peak powers of $3-30 \mathrm{~kW}$ assuming a pulse duration of $1 \mathrm{ps}$. 


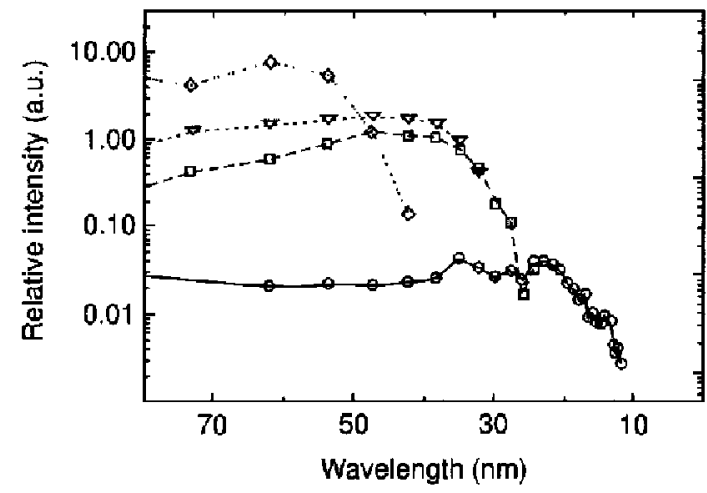

Figure 12 Harmonic intensities of Ti:sapphire $(810 \mathrm{~nm}, \sim 50 \mathrm{~mJ})$ radiation for different noble gases: $\vartheta$, xenon; $\nabla$, krypton; $\square$, argon; $O$, neon.

argon, this result is in good agreement with detailed theoretical and experimental investigations from Wahlström and L'Huillier, respectively [22, 24]. The observed cutoff for neon is not real and is caused by the strong decrease of the sensitivity of the detection system at wavelengths below $12 \mathrm{~nm}$. For helium as nonlinear medium the intensity of the harmonics was too weak for an unambiguous separation from noise.

Absolute photon numbers have been measured for the harmonics of the excimer laser radiation using a calibrated XUV semiconductor photodiode (UDT X-UV 20). The stated sensitivity of the diode agrees in the visible spectral range within a factor of 2 with measured values, using subpicosecond pulses at $496 \mathrm{~nm}$.

As shown in Fig. 11, at the third harmonics of the $\mathrm{KrF}$ system, photon numbers of the order of $10^{9}$ to $10^{10}$ have been measured. For the Titsapphire laser such absolute measurements have not been performed until now, but in [22] photon numbers of $10^{8}-10^{9}$ at $60 \mathrm{~nm}$ for optimum conditions (long confocal parameter) have been determined, which are about one order of magnitude smaller than for the $\mathrm{KrF}$ pump radiation for our experimental conditions. However, at wavelengths below about $20 \mathrm{~nm}$, harmonics from the longer-wavelength lasers seem to be more powerful [27].

The present power of the harmonics already corresponds to peak brightnesses which are several orders of magnitude higher than those of synchrotron sources in this spectral range [28]. Therefore, high harmonics may be an alternative, compact, and easy to realize source, especially for those applications that require high peak powers of short pulses.

\section{Generation of tunable XUV radiation by high-order frequency mixing}

For many applications tunable XUV radiation is desirable and, in principle, can be realized by the harmonic generation process if tunable pump lasers are used. Among the high peak power sources, only Ti:sapphire is sufficiently tunable. However, tuning of a terawatt laser system as shown in Fig. 3 is difficult, because the oscillator and the whole pulse chirping and compression units have to be adjusted. Therefore, it is more practicable to mix the radiation of a high-power fixed-frequency laser system $\left(\omega_{\mathrm{p}}\right)$ with a less powerful but easily tunable light source $\left(\omega_{\text {tun }}\right)$ to generate tunable radiation at $n \omega_{\mathrm{p}} \pm k \omega_{\text {tum }}(n+k$ odd) by high-order frequency mixing. Such mixing experiments have been performed with the ArF and $\mathrm{KrF}$ excimer radiation and the $\mathrm{KrF}$ and $496 \mathrm{~nm}$ dye laser radiation at fixed frequencies, and with the Ti:sapphire laser and a tunable OPG system to generate tunable XUV radiation.

The experimental setup for the frequency mixing experiments is similar to that for harmonic 

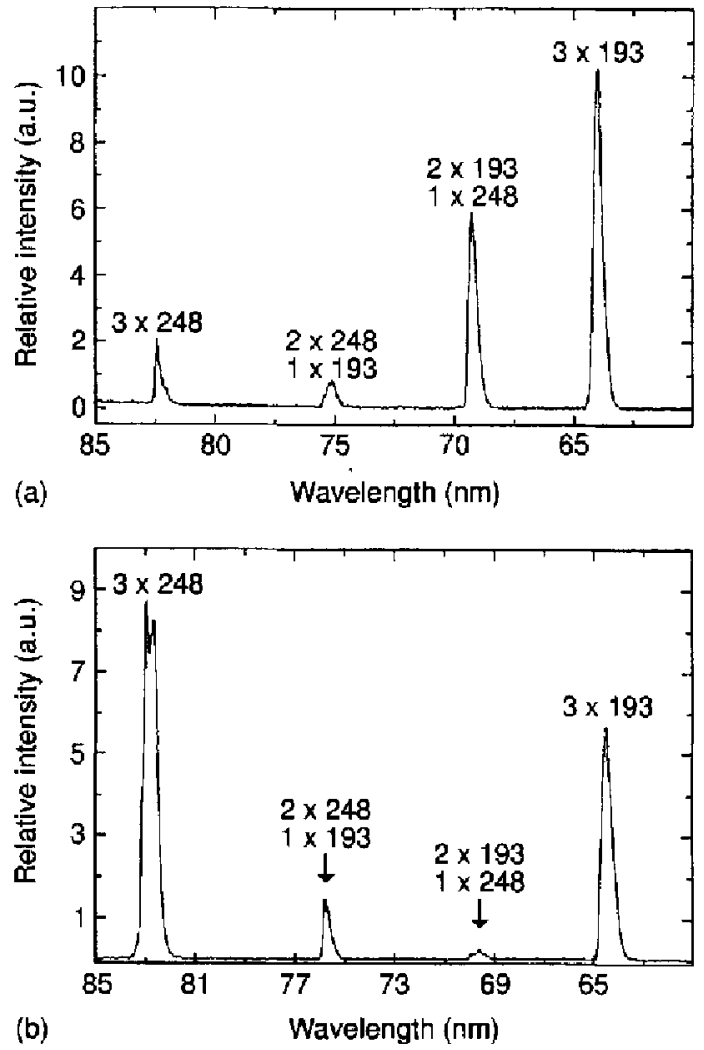

Figure 13 Third-order mixing spectrum of KrF (248 $\mathrm{nm}$ ) and ArF $(193 \mathrm{~nm})$ radiation in $\mathrm{H}_{2}$ (a) and argon (b). Pump intensities $\sim 10^{15} \mathrm{~W} \mathrm{~cm}^{-2}$. The relative intensity scales are comparable.

generation (Fig. 7), except that two laser fields have to be overlapped spatially and temporally in the nonlinear medium, which was accomplished by focusing one beam through a dichroic mirror and by a variable delay line.

Figure 13 shows as an example third-order mixing of $\mathrm{KrF}(248 \mathrm{~nm})$ and $\operatorname{ArF}(193 \mathrm{~nm})$ short pulses in argon and molecular hydrogen at comparable focused intensities $\left(10^{15} \mathrm{~W} \mathrm{~cm}^{-2}\right)$. Here, the influence of multiphoton resonances can be observed. Both the third harmonic of ArF $(193 \mathrm{~nm})$ and the $2 \times 193 \mathrm{~nm}+248 \mathrm{~nm}$ mixing signals are strongly increased in the case of hydrogen compared to argon, whereas the third harmonic of the $\mathrm{KrF}$ laser is much stronger in argon compared to hydrogen. This can be explained by a strong two-photon resonance for the $193 \mathrm{~nm}$ radiation in molecular hydrogen $\left(\mathrm{X}^{1} \Sigma_{\mathrm{g}}^{+}(\nu=0)-\mathrm{E}, \mathrm{F}^{1} \Sigma_{\mathrm{g}}^{+}(\nu=2)\right)$ [29] and a three-photon resonance enhancement for the $248 \mathrm{~nm}$ radiation in argon $\left(3 \mathrm{p}^{6} \mathrm{~S}_{0}-4 \mathrm{~d}[3 / 2]_{1}^{0}\right)$ [30].

In Fig. 14 all fifth-order sum mixing signals of ArF and $\mathrm{KrF}$ laser radiation and in Fig. 15 seventh-order mixing signals of $\mathrm{KrF}$ radiation with $496 \mathrm{~nm}$ radiation internally generated in the KrF system (see Fig. 2) are shown. The spectra clearly indicate that tunable XUV radiation can be generated if one of the mixing fields is tunable. The $\mathrm{KrF} 248 \mathrm{~nm} / 496 \mathrm{~nm}$ mixing signals could be detected down to about $10 \mu \mathrm{J}$ of the $496 \mathrm{~nm}$ radiation at intensities around $10^{12} \mathrm{~W} \mathrm{~cm}^{-2}$. In order to realize broadly tunable XUV radiation by this mixing concept, a tunable short-pulse source with energies of at least several bundred microjoules, allowing intensities of more than $10^{13} \mathrm{~W} \mathrm{~cm}^{-2}$, is needed. For this, we have developed an optical parametric 

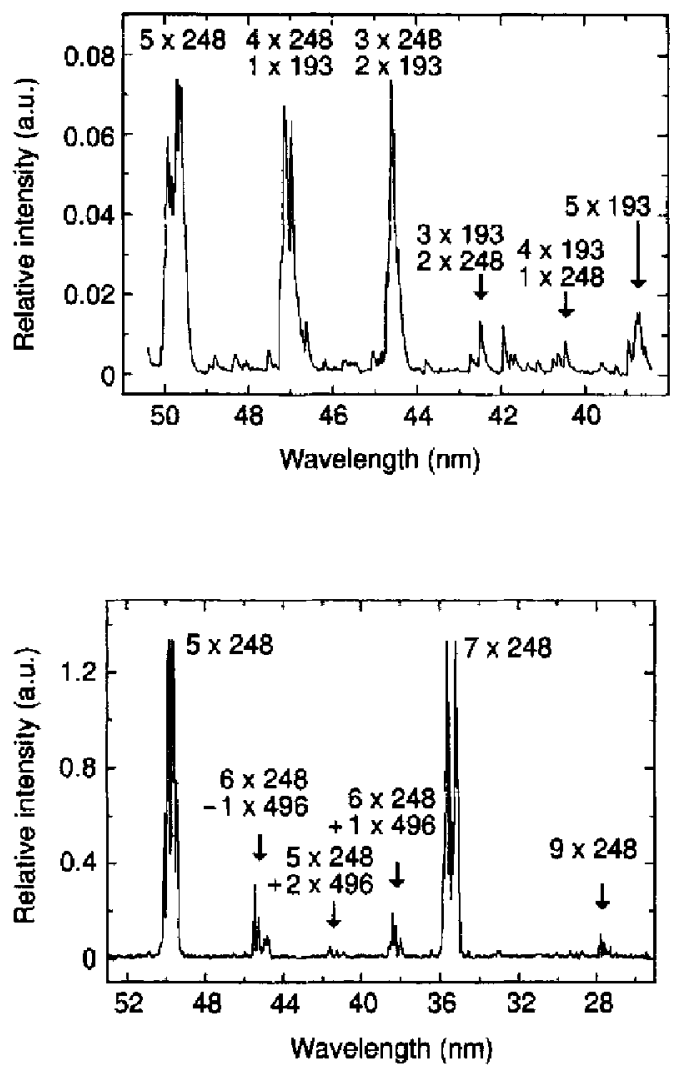

Figure 14 Fifth-order harmonic and mixing spectrum of $\mathrm{KrF}(248 \mathrm{~nm})$ and $\mathrm{ArF}(193 \mathrm{~nm})$ radiation in argon. Pump intensities $\sim 10^{15} \mathrm{~W} \mathrm{~cm}^{-2}$. Scale comparable to relative scale of Fig. 13. The modulation of the intensity of the signals is mainly due to pulse-to-pulse fluctuations of the lasers (no singleshot spectrum).

Figure 15 Seventh-order harmonic and mixing spectrum of $248 \mathrm{~nm}\left(\sim 5 \times 10^{15} \mathrm{Wcm}^{-2}\right)$ and $496 \mathrm{~nm}\left(\sim 10^{13} \mathrm{~W} \mathrm{~cm}^{-2}\right)$ radiation in helium. Scale comparable to scales of Figs 13 and 14. The modulation of the intensity of the signals is mainly due to pulse-to-pulse fluctuations of the lasers (no singleshot spectrum).

generator-amplifier (OPG) source pumped by frequency-doubled Ti:sapphire laser radiation and have mixed radiation from this source with the fundamental of the Ti:sapphire system (Fig. 3).

The setup is shown in Fig. 16. With about $5-10 \mathrm{~mJ}(\sim 200 \mathrm{fs})$ at $405 \mathrm{~nm}$, some $300-400 \mu \mathrm{J}$ ( $600 \mathrm{fs}$ ) of OPG radiation, so far tunable in the range $520-650 \mathrm{~nm}$, could be generated. With this tunable radiation, frequency mixing signals ( $2 n \omega_{\mathrm{p}} \pm \omega_{\mathrm{OPG}}$; in first order of the OPG radiation) could be resolved up to wavelengths of $46 \mathrm{~nm}$ in xenon and $37 \mathrm{~nm}$ in argon, as shown in Fig. 17. For xenon also weak signals at $(2 n+1) \omega_{\mathrm{p}} \pm 2 \omega_{\mathrm{OPG}}$ (second order of the OPG radiation) could be detected [31]. Figure 18 shows in more detail a detuning of the ninth-order sum mixing signal in xenon around $87 \mathrm{~nm}$. As discussed in [31], with the first- and second-order OPG mixing process and the potential tuning range of the OPG signal wave (about $480 \mathrm{~nm}$ to $800 \mathrm{~nm}$ ), practically the whole range between two subsequent harmonics of the Ti:sapphire radiation can be covered, so that in principle tunable short-pulse radiation down to the highest-order harmonics in neon $(\sim 10 \mathrm{~nm})$ should also be possible if the intensity of the OPG can be further increased by increasing the output energy and by improving the beam quality.

\section{Conclusions}

In recent years, nonperturbative high-order harmonic generation has been rigorously investigated, and short wavelengths as well as good output powers have been achieved. Recently 


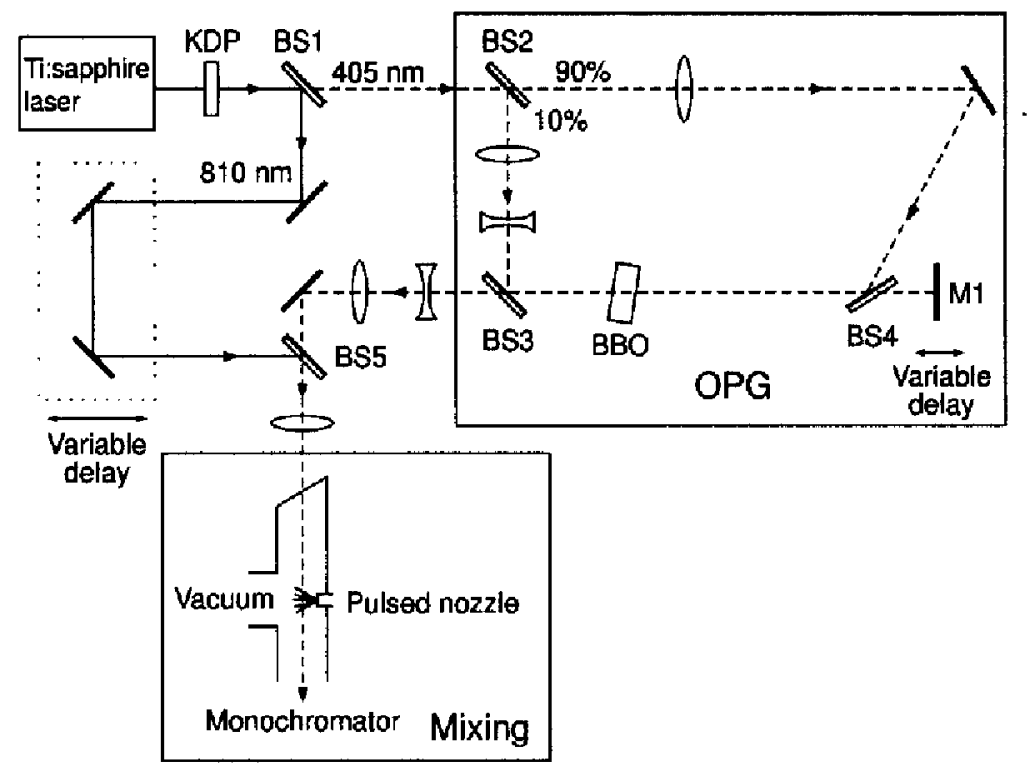

Figure 16 Setup for high-order frequency mixing of Ti:sapphire $(810 \mathrm{~nm})$ radiation with tunable radiation from an optical parametric generator-amplifier (OPG). Pump radiation at $405 \mathrm{~nm}$ is used to generate parametric fluorescence in BBO. At mirror $\mathrm{M} 1$ back-reflected fluorescence is used as seed radiation in a second amplification pass through the BBO crystal.

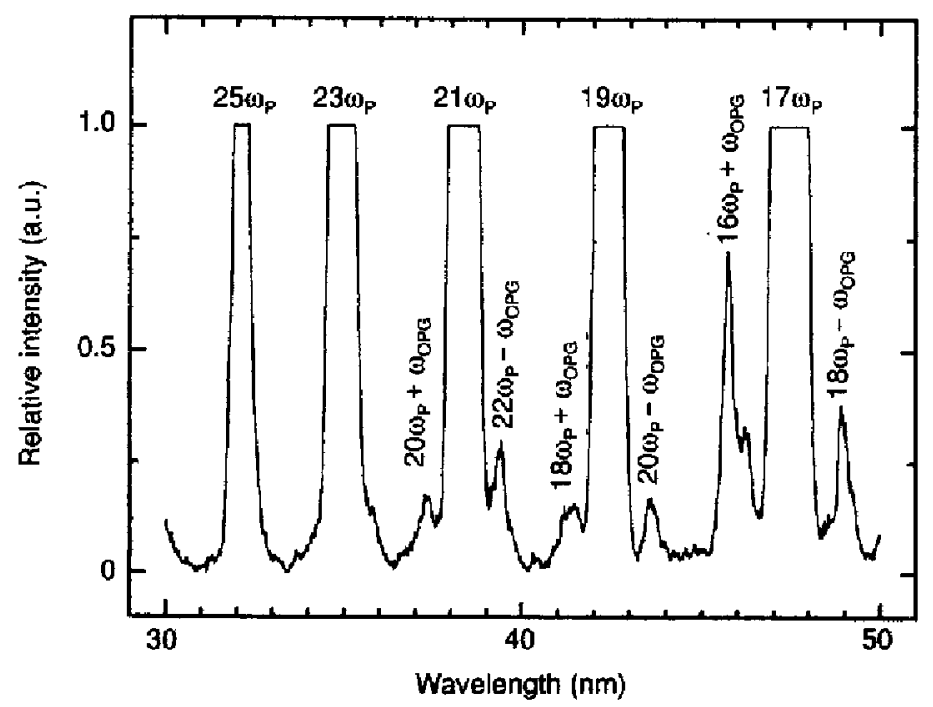

Figure 17 Part of the high-order harmonic and mixing spectrum of Tisapphire $\left(\omega_{\mathrm{p}} 810 \mathrm{~nm}, \sim 10^{15} \mathrm{~W} \mathrm{~cm}^{-2}\right)$ and $O P G\left(\omega_{\mathrm{OPG}} 520-650 \mathrm{~nm}, \leq 400 \mu\right)$ radiation in argon. 


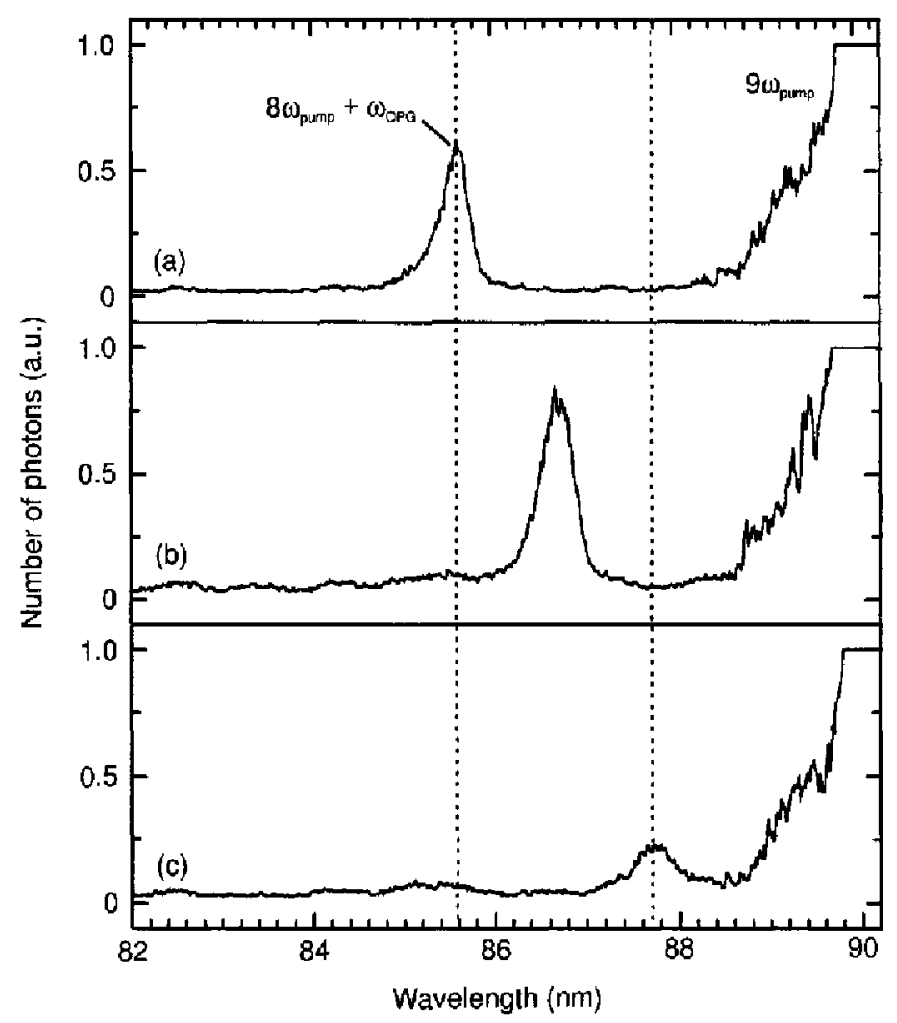

Figure 18 Demonstration of tunability of the $8 \omega_{\text {purnp }}+\omega_{\text {OPG }}$ mixing signal in the vicinity of the ninth-order Ti:sapphire harmonic for xenon as nonlinear material. The OPG radiation has been tuned to three different wavelengths: (a) $545 \mathrm{~nm}$; (b) $590 \mathrm{~nm}$; (c) $640 \mathrm{~nm}$.

first applications of these sources have also been reported or planned in spectroscopy [32] and as seed radiation in $\mathrm{x}$-ray laser research.

In this paper low- and higher-order mixing schemes have been described that allow tunability in principle down to the shortest wavelengths achieved with the harmonics. This will stimulate further applications.

By four-wave mixing in xenon, tunable short-pulse VUV radiation with peak powers approaching the gigawatt range has been realized, which should allow novel nonlinear experiments.

For the higher-order mixing process, the power generated is still small, but can surely be significantly improved by higher pump intensities and the optimization of focusing and phasematching conditions. For two-colour mixing experiments additional degrees of freedom for influencing the process exist. In the first $\omega-2 \omega$ mixing experiments we investigated the intensities of the mixing signals and the position of the cutoff for linear and circular polarizations and different relative orientations of the two fields [33].

Finally, considering effort and progress in the realization of compact high-power laser systems, it can be expected that the generation of coherent XUV radiation by nonlinear optics will develop into a standard laboratory tool. 


\section{Acknowledgements}

The authors thank $H$. Jacobs for valuable help in preparing the manuscript and the Deutsche Forschungsgemeinschaft for financial support of the project.

\section{References}

1. Procedings of the 3rd International Colloquium on X-Ray Lasers 1992, Schliersee, Germany, 1992, cdited by E. E. Fill, Institute of Physics Conference Series 125.

2. R. C ELTON, X-Ray Lasers (Academic Press, New York, 1990).

3. I. REINTJES, C. Y. SHI and R. C. ECKARDT, IEEE J. Quantum Electron. QE-14 (1978) 581.

4. C E. M STRAUSS and D. J. FUNK, Opt. Lett. 16 (1991) 1192.

5. Y. HIRAKAWA, T. OKADA, M. MEODA and K MLRAOKA, Opt. Commun. 84 (1991) 365.

6. G. HILBER, A. LAGO and R. WALLENSTEIN, . Opt. Soc. Am. $B 4$ (1987) 1753.

7. P C. HINNEN, W. HOGERVORST, S. STOLTE and W. UBACHS, Appl. Phys. $\boldsymbol{B} 59$ (1994) 307.

8. K. S. E. EIKEMA, W. UBACHS, W. VASSEN and W. HOGERVORST, Phys. Rev. Lett. 68 (1993) 1690.

9. A. LHUILLIER and PH. BALCOU, Phys. Rew. Lett. 70 (1993) 774.

10. J. J. MACKLIN, J. D KMETEC and C. L GORDON, Phys. Rev. Lett. 70 (1993) 766.

11. K. MIYAZAKI and H. SAKaI, d. Phys. B: At. Mol. Opt. Phys. 25 (1992) L83.

12. N. SARUKURA, K. HATA. T. ADACHI, R. NODOMI, M. WATANABE and S. WaTANaBe. Phys. Rev. A 43 (1991) 1669.

13. M. D. PERRY and G. MOUROL, Science 264 (1994) 917.

14. A. TUNnermanN, K. MOSSAvi and B. Wellegehalsen, Phys. Rev. A 46 (1992) 2707

15. S. SZATMÁRI and F. P. SCHÁFER, Opt. Commun. 68 (1988) 196.

16. G. ALMÁSI, S. SZATMÁRI and F. SIMON, Opt. Commun. 88 (1992) 231.

17. A. TUNNERMANN, C MOMMA, K. MOSSAVI, C. WINDOLPH and B. WELEEGEHALSEN, IEEE J. Quantum Electron. QE-29 (1993) 1233.

18. C. MOMMA, H. EICHMANN, K. MOSSAVI, M. FEUERHAKE and B. WELLEGEHAUSEN, Proceedings of the VIIth International Symposium on Ultrafast Processes in Spectroscopy. Vilnius, Lithuania, 1993, p. 339.

19. C. MOMMA, H. EICHMANN. H. JACOBS, A. TUNNERMANN, H. WELLING and B. WELLEGEHAUSEN, Opt. Lett. 18 (1993) 516.

20. C. MOMMA, H. EICHMANN, A TĹNNERMANN, P. SIMON, G. MAROWSKY and B. WELLEGEHAUSEN, Opt. Lett. 18 (1993) 1180.

21. J. L. KRAUSE, K. J. SCHAFER and K. C. KULANDER, Phys. Rev. Lett. 68 (1992) 3535.

22. C.-G. WAHLSTROM, J. LARSSON, A. PERSSON, et al., Phys, Rev. A 48 (1993) 4709.

23. W. BECKER, S. LONG and J. K. MeIVER, Phys. Rev. A 50 (1994) 1540.

24. A. L'HUILIIER, M. LEWEN\$TEN, P. SALIERES, et al., Phys. Rev. A 48 (1993) R3433.

25. T. SRINIVASAN, K BOYER, H. EGger, et al, Picosecond Phenomena $I I$, edited by K. B. Eisenthal, R. M. Hochstrasser, W. Kaiser and A. Laubereau (Chemical Physics 23; Springer, Berlin, 1982).

26. L. V. KELDYSH, Sov. Phys. JETP 20 (1965) 1307.

27. K. KONDO, N. SaRUKURA, K SaJIKI and S. WATANABE, Phys. Rev. A 47 (1993) R2480.

28. M. H. KEY, Nature 316 (1985) 314.

29. H. PUMMER, T SRINIVASAN, H. EGGER, T. S. LUK and C. K. RHODES, Opt. Lett. 7 (1982) 93.

30. R. L. KELLY, I, Phys. Chem. Ref. Data 16 (Supplement 1) (1987) 42.

31. H. EICHMANN, S. MEYER, K. RIEPL, C. MOMMA and B. WELLEGEHAUSEN, Phys. Rev. A S0 (1994) R2834.

32. R. HAIGHT and P. F. SEIDLER, Appl. Phys. Lett. 65 (1994) 517.

33. H. EICHMANN, A. EGBERT, S. NOLTE, et al, Phys. Rev. A 51 (1995) R3414. 